\title{
Papers
}

\section{Reduction in mortality after inappropriate early discharge from intensive care unit: logistic regression triage model}

Topic: 21;118;218

Kathleen Daly, R Beale, R W S Chang

\begin{abstract}
Objective To develop a predictive model to triage patients for discharge from intensive care units to reduce mortality after discharge.

Design Logistic regression analyses and modelling of data from patients who were discharged from intensive care units.

Setting Guy's hospital intensive care unit and 19 other UK intensive care units from 1989 to 1998. Participants 5475 patients for the development of the model and 8449 for validation.

Main outcome measures Mortality after discharge and power of triage model.

Results Mortality after discharge from intensive care was up to $12.4 \%$. The triage model identified patients at risk from death on the ward with a sensitivity of $65.5 \%$ and specificity of $87.6 \%$, and an area under the receiver operating curve of 0.86 . Variables in the model were age, end stage disease, length of stay in unit, cardiothoracic surgery, and physiology. In the validation dataset the $34 \%$ of the patients identified as at risk had a discharge mortality of $25 \%$ compared with a $4 \%$ mortality among those not at risk.

Conclusions The discharge mortality of at risk patients may be reduced by $39 \%$ if they remain in intensive care units for another 48 hours. The discharge triage model to identify patients at risk from too early and inappropriate discharge from intensive care may help doctors to make the difficult clinical decision of whom to discharge to make room for a patient requiring urgent admission to the unit. If confirmed, this study has implications on the provision of resources.
\end{abstract}

\section{Introduction}

The winter of 1999 highlighted the acute shortage of intensive care beds in the United Kingdom. A consequence of shortage is that patients are often discharged early and perhaps inappropriately to make room for more severely ill patients. A study in 1993 reported mortality after discharge from intensive care from $6.1 \%$ to $16.3 \% .^{12}$ The causes of death after such discharge may be due to factors occurring before ${ }^{34}$ or after discharge..$^{5-7}$ Goldfrad and Rowan, who used discharges at night as a proxy measure of inappropriate early discharge from intensive care, reported a 1.4- fold increase in ultimate hospital mortality among patients discharged at night. ${ }^{8}$ Patients who died after discharge had significantly higher severity of illness scores or therapeutic intervention scores on the day of discharge than those who survived. ${ }^{910}$

We report on the development of a predictive triage model for discharge to identify patients at risk of dying after discharge from intensive care. We also explored the implications of its use.

\section{Methods}

We included in the study all patients discharged from the 13 bed intensive care unit at Guy's hospital between 1 June 1990 and 31 December 1998 and from 19 UK units (Riyadh ICU program users group, RIPUG) between June 1989 and September 1996. We analysed daily physiological and treatment data collected prospectively through the Riyadh ICU program (Medical Associated Software House, London) to identify candidate variables for the model. We measured severity of illness and intensity of treatment with the acute physiology and chronic health evaluation II (APACHE II) system, ${ }^{11}$ the organ failure score,${ }^{12}$ and the therapeutic intervention scoring system. ${ }^{13}$ These data, together with demographic data including the presence of chronic ill health (as defined with APACHE II criteria) and patients' hospital outcome, were entered daily on to the computer by a team of specifically trained nurses and doctors.

In general, patients were considered for admission to the participating intensive units if the combined assessment of the referring clinician and the doctor in charge of the unit were that the patient would benefit from intensive care. Clinical judgment on the basis of physiological variables, concurrent treatment, and clinical assessment was used to discharge patients from the intensive care unit. When there is pressure on beds, the least ill patient who can be managed outside an intensive care unit (for example, without mechanical ventilation) would be considered for discharge from the unit. None of the 20 units had a high dependency unit during this study.

\section{Model development}

There were 6319 patients admitted to the 13 bed general (medical, surgical, and cardiothoracic) adult intensive care unit at Guy's hospital between 30 June 1990

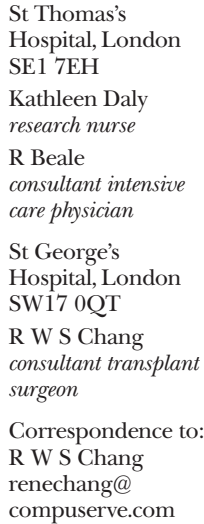

BMJ 2001;322:1-6 
Table 1 Sensitivity and specificity (with cut offs of $0.5,0.6$, and 0.7 ), receiver operator characteristic curve (ROC) analysis, and Hosmer-Lemeshow statistic produced from each of 20 data subsets

\begin{tabular}{|c|c|c|c|c|c|c|c|c|}
\hline \multirow[b]{2}{*}{ Model } & \multicolumn{3}{|c|}{ Sensitivity (No of deaths correct/200 patients) } & \multicolumn{3}{|c|}{ Specificity (No of survivors correct/250 patients) } & \multirow[b]{2}{*}{ ROC (\%) } & \multirow[b]{2}{*}{$\chi^{2}$; P value } \\
\hline & 0.5 & 0.6 & 0.7 & 0.5 & 0.6 & 0.7 & & \\
\hline 1 & $70.5(141)$ & $57.0(114)$ & $44.5(89)$ & $80.0(200)$ & 86.4 (216) & $92.4(231)$ & 83.0 & $3.85 ; 0.87$ \\
\hline 2 & $71.0(142)$ & 60.5 (121) & $48.0(96)$ & 79.2 (198) & 87.2 (218) & $93.6(234)$ & 83.7 & $4.86 ; 0.77$ \\
\hline $3^{*}$ & $69.0(138)$ & $54.0(108)$ & $33.5(67)$ & $71.2(178)$ & $82.8(207)$ & $92.0(230)$ & 79.5 & $13.30 ; 0.10$ \\
\hline 4 & 76.0 (152) & $62.0(124)$ & $51.0(102)$ & 80.4 (201) & 87.2 (218) & $92.4(231)$ & 84.7 & $4.22 ; 0.84$ \\
\hline 5 & $74.0(148)$ & $65.0(130)$ & $49.5(99)$ & $81.2(203)$ & $87.2(218)$ & $92.0(230)$ & 84.8 & $5.68 ; 0.68$ \\
\hline $6^{*}$ & 72.0 (144) & 60.5 (121) & $48.5(97)$ & $80.0(200)$ & $88.0(220)$ & $92.8(232)$ & 83.9 & $7.26 ; 0.51$ \\
\hline 7 & 72.5 (145) & 61.0 (122) & $48.5(97)$ & 82.0 (205) & $86.8(217)$ & $91.6(229)$ & 84.0 & $7.71 ; 0.46$ \\
\hline 8 & $73.0(146)$ & 61.5 (123) & 50.5 (101) & 83.6 (209) & $88.0(220)$ & $92.8(232)$ & 85.2 & $6.82 ; 0.56$ \\
\hline $9^{*}$ & $73.0(146)$ & 61.5 (123) & $42.5(85)$ & 78.4 (196) & $84.0(210)$ & $91.6(229)$ & 82.0 & $4.96 ; 0.76$ \\
\hline 10 & $73.0(146)$ & 64.0 (128) & 55.0 (110) & $83.2(208)$ & $87.6(219)$ & $94.0(235)$ & 82.0 & $6.6 ; 0.58$ \\
\hline 11 & 76.5 (153) & 66.0 (132) & 54.0 (108) & 82.4 (206) & $85.6(214)$ & $91.2(228)$ & 85.2 & $11.08 ; 0.2$ \\
\hline 12 & 70.5 (141) & $57.0(114)$ & $44.5(89)$ & $80.0(200)$ & $86.4(216)$ & 92.4 (231) & 82.3 & $3.85 ; 0.87$ \\
\hline $13^{*}$ & $73.0(146)$ & 62.5 (125) & $44.0(88)$ & 78.4 (196) & $85.6(214)$ & $92.8(232)$ & 82.5 & $6.02 ; 0.64$ \\
\hline 14 & 75.5 (151) & 61.0 (122) & 51.5 (103) & 80.4 (201) & $86.4(216)$ & $91.6(229)$ & 84.9 & $4.23 ; 0.84$ \\
\hline 15 & $72.0(144)$ & 58.5 (117) & $47.0(94)$ & 78.4 (196) & $86.8(217)$ & $92.4(231)$ & 82.7 & $4.08 ; 0.85$ \\
\hline 16 & $72.0(144)$ & $57.0(114)$ & $46.5(93)$ & 80.4 (201) & $86.4(216)$ & $92.4(231)$ & 82.8 & $2.75 ; 0.95$ \\
\hline 17 & 76.5 (153) & 65.5 (131) & 56.0 (112) & 80.8 (202) & $88.0(220)$ & $92.0(230)$ & 82.8 & $8.95 ; 0.35$ \\
\hline $18 \dagger$ & $73.0(146)$ & $65.5(130)$ & 52.0 (104) & $82.4(206)$ & $87.6(219)$ & $93.6(234)$ & 85.6 & $5.92 ; 0.66$ \\
\hline 19 & $70.5(141)$ & $58.0(116)$ & $46.0(92)$ & 78.0 (195) & 84.4 (211) & $92.0(230)$ & 82.4 & $8.83 ; 0.36$ \\
\hline $20^{*}$ & 76.0 (152) & $65.5(131)$ & $53.0(106)$ & $80.4(201)$ & $87.2(218)$ & $93.2(233)$ & 85.1 & $2.56 ; 0.96$ \\
\hline
\end{tabular}

*Data subsets did not include all five variables selected in other 15 data subsets. †Model used for validation study.

and 31 December 1996. We excluded from the analysis the $844(13.4 \%)$ patients who died on the unit. Of the $5475(87.0 \%)$ survivors, $200(3.7 \%)$ patients died on the wards and $5275(96.3 \%)$ survived to leave hospital. Twenty five $(12.5 \%)$ patients who died on the ward and $117(2.2 \%)$ hospital survivors were readmitted to intensive care during the same hospital stay. Only data from the patient's last day (the day of discharge or the day immediately preceding discharge; the last day with at least 8 hours of data) in the unit during their first admission to intensive care were used to develop the predictive model. There were 3133 (57.2\%) patients who were admitted to intensive care after cardiothoracic surgery ( $97 \%$ after elective surgery) - a relatively low risk group. We created a variable denoting whether or not the patient had undergone cardiothoracic surgery (code 1 and 0 , respectively).

We used univariate analysis to identify candidate variables for the model. Variables with a significant influence on survival $(\mathrm{P}<0.05)$ after discharge from intensive care were subjected to multivariate logistic modelling as linear effects with the binary logistic regression model (SPSS, Woking). A stepwise forward logistic regression procedure was used to derive the model. The initial attempts with model building were not successful, possibly because of loss of discriminatory power of the logistic regression function because of the large disparity in the number of survivors compared with non-survivors. To correct for this, we merged a random selection of 250 cases from the 5275 hospital survivors with the 200 ward deaths to obtain a dataset for model development. (This was a rather unconventional approach borrowed from the training of neural networks that commonly uses data equalisation. ${ }^{14}$ ) We repeated this 20 times to produce 20 modelling datasets; the 250 randomly selected cases were replaced before we selected another 250 cases (table 1 ). We then selected the "best" model (largest area under the receiver operator characteristic curve) and cut off (best trade off between sensitivity and specificity). Cali- bration of the model was assessed by the HosmerLemeshow "goodness of fit statistic" 15 for significance $(\mathrm{P}>0.05)$. We assessed discrimination with receiver operating curve analysis. ${ }^{16}$

\section{Model validation}

We evaluated the triage model by applying it to a different dataset, derived from 1136 survivors $(84.3 \%$ of admissions) from the intensive care unit at Guy's hospital who had been admitted between 1 January 1997 and 31 December 1998 and 7313 survivors (76.6\% of admissions) from 19 other UK units (Riyadh ICU program users group) who had been admitted between June 1989 and September 1996. We used a new dataset for validation of the model to avoid any overoptimistic findings that may have occurred had we used the development dataset. ${ }^{17}$ Furthermore, as the development dataset contained many patients who had undergone cardiac surgery we considered it important to evaluate the model's validity among units in which this was not the case.

\section{Use of model to alter outcome}

For the model to be of any use we must be able to affect the outcome of patients identified as at risk. To test this, we selected patients who had stayed in intensive care for more than three days and had been at risk of death at any time within the 48 hours before discharge from the unit. We excluded from analysis those patients who died on the ward and who had been classified as "not for resuscitation" at discharge from intensive care because in real time it would not make any sense to prolong the stay in intensive care of these patients. The patients were classified into four subgroups according to the timing of the prediction of risk relative to their discharge from the unit. Group 0 comprised patients predicted to be at risk on the day of discharge; group 1 comprised patients predicted to be at risk in the 24 hours before discharge; group 2 comprised those predicted to be at risk in the 48 hours before discharge; and group 3 comprised patients who were not at risk in 
Table 2 Demographic characteristics and clinical features of survivors of intensive care for three datasets. Figure are number (percentage) of patients, median (range), or mean (SD)

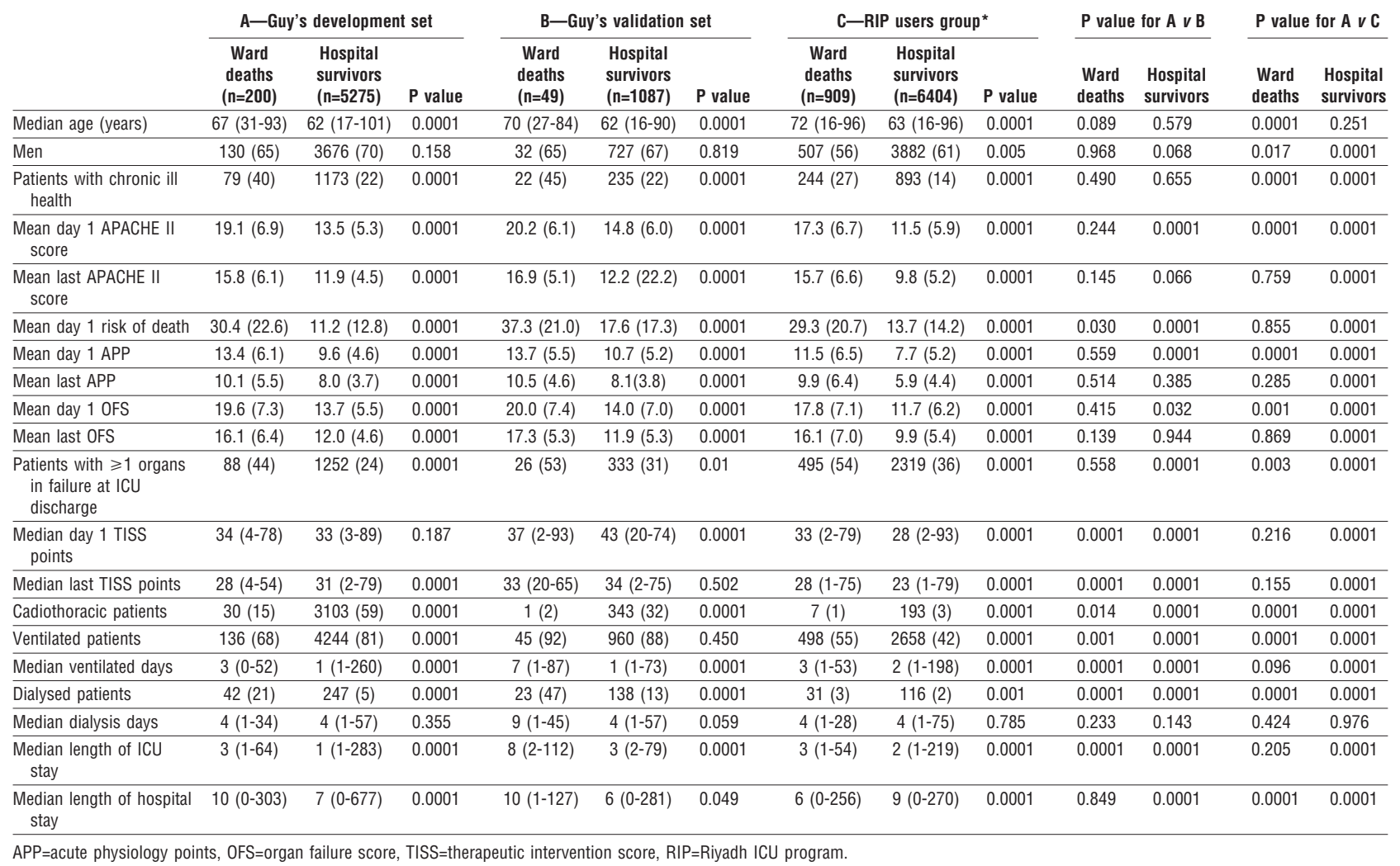

the 48 hours before discharge. Groups 1, 2, and 3 were not at risk on day of discharge.

Data analysis was performed with the statistical software package SPSS version 9.0. Categorical data were analysed with $\chi^{2}$ tests. Non-normally distributed continuous data were evaluated with the MannWhitney test. Logistic regression analysis was used to develop the predictive model. $\mathrm{P}<0.05$ was considered significant.

This study was approved by the local ethics committee of Guy's Hospital.

\section{Results}

Table 2 gives demographic data and details of clinical features, severity of illness, and candidate variables for the model. The following variables were considered in the models: acute physiology points, length of stay in intensive care, therapeutic intervention score, duration (days) on mechanical ventilation, dialysis, age, presence of chronic ill health, number of failing organs, and whether or not the patient had had cardiothoracic surgery. Acute physiology points was used in preference to APACHE II score as the latter is derived from the acute physiology points, age points, chronic ill health points, and presence or absence of emergency surgery.

Forward stepwise multivariate analyses on each of the 20 modelling datasets (table 1) selected the following five variables: patient's age, chronic health points, acute physiology points at discharge from unit, length of stay in unit, and whether or not the patient had had cardiothoracic surgery for inclusion in the model in 15 instances. A cut off of 0.6 gave the best sensitivity and specificity (65.5\% and $87.6 \%$, respectively, in model 18 ). Table 3 gives details of the final model, and figure 1 shows its receiver operating curve.

As the results of the two validation datasets were similar we merged the data (table 4). The sensitivity and specificity were $74.3 \%$ and $71.1 \%$, respectively; and the area under the receiver operator characteristic curve (fig 2) was 0.80 (95\% confidence interval 0.79 to 0.81 ). The area under the curve ranged from 0.68 to 0.87 for the 20 individual intensive care units. Mortality in patients identified as at risk was $25 \%$ while the mortality in those not at risk was $4 \%$, giving a relative risk of 5.61 (4.89 to 6.44). In the development dataset the figure for relative risk was 9.44 (7.12 to 21.51).

There were significant differences in mortality after discharge from intensive care between groups 0,1 , and 2 (table 5). In the development dataset, $14 \%$ of at risk

Table 3 Six variables selected by forward stepwise multiple logistic regression analysis for discharge triage predictive model ${ }^{\star}$

\begin{tabular}{|c|c|c|}
\hline Variable & $\beta$ (SE) & $P$ value \\
\hline Age & $0.0532(0.0094)$ & $<0.0001$ \\
\hline Chronic health points & $0.2501(0.0728)$ & 0.0006 \\
\hline Acute physiology points & $0.1556(0.0300)$ & $<0.0001$ \\
\hline Cardiac surgery & $-2.1084(0.2712)$ & $<0.0001$ \\
\hline Length of ICU stay & $0.0447(0.0153)$ & 0.0034 \\
\hline Constant & $-4.5821(0.6825)$ & $<0.0001$ \\
\hline
\end{tabular}


Table 4 Predictive power of discharge triage model. Figures are number (percentage) of patients

\begin{tabular}{lccc} 
Sensitivity/specificity cut off point & Alive & Died & Total \\
\hline Development dataset & & & \\
\hline$\geqslant 0.6$ & $770(86)$ & $130(14)$ & 900 \\
\hline$<0.6$ & $4505(98.5)$ & $70(1.5)$ & 4575 \\
\hline Combined validation datasets & & & \\
\hline$\geqslant 0.6$ & $2163(75)$ & $712(25)$ & 2875 \\
\hline$<0.6$ & $5328(96)$ & $246(4)$ & 5574 \\
\hline
\end{tabular}

Table 5 Comparison of mortality after discharge from intensive care for patients discharged on day of prediction of risk (group 0), patients who stayed additional 24 hours (group 1), patients who stayed additional 48 hours (group 2), and patients at no risk (group 3). Mortality is reduced by $39.3 \%$ if patients stay another two days. Figures are number (percentage) of patients

\begin{tabular}{|c|c|c|c|}
\hline & Alive & Died & Total \\
\hline \multicolumn{4}{|c|}{ Development dataset ${ }^{\star}$} \\
\hline Group 0 & $326(86)$ & $53(14)$ & 379 \\
\hline Group 1 & $71(93)$ & $5(7)$ & 76 \\
\hline Group 2 & $52(96)$ & $2(4)$ & 54 \\
\hline \multicolumn{4}{|c|}{ Combined validation dataset $\dagger$} \\
\hline Group 0 & $581(72)$ & $230(28)$ & 811 \\
\hline Group 1 & $126(87)$ & $19(13)$ & 145 \\
\hline Group 2 & $86(83)$ & 17 (17) & 103 \\
\hline Group 3 & $776(96)$ & $34(4)$ & 810 \\
\hline
\end{tabular}

patients died on the ward. In at risk patients who stayed an additional 48 hours in intensive care, during which time the probability of dying fell below 0.6 , mortality after discharge from intensive care was only $4 \%$ $(\mathrm{P}=0.034)$. The relative risk of mortality for groups 1 and 2 versus group 0 (discharged on the day risk was predicted) was 0.385 (0.18 to 0.826 ). In the validation dataset there was a reduction in mortality from $28 \%$ in group 0 to $17 \%$ among those who stayed another 48 hours $(\mathrm{P}=0.011)$-that is, their relative risk was reduced from 6.76 (4.87 to 9.56) in group 0 versus group 3 to 3.46 (2.21 to 5.41) in group 1 and 2 versus group 3 . The relative risk of mortality for those who stayed an additional 24 and 48 hours compared with group 0 was 0.512 (0.373 to 0.706$)$.

\section{Potential impact on the provision of intensive care beds}

We used the validation dataset to estimate the impact on the provision of intensive care resources. There were 8449 patients who stayed in intensive care for a total of 34588 days, with an overall mortality after discharge from intensive care of $11.3 \%$. We identified 2875 patients ( $34 \%$ of total) as at risk, with a mortality after discharge of $25 \%$. If we assume that our model is valid, mortality after discharge from intensive care could be reduced by nearly $39 \%$ if these patients stayed another two days before discharge. We estimated that this would required 5750 additional intensive care bed days or the provision of fully staffed intensive care bed days would have to be increased by $16 \%$.

\section{Discussion}

A considerable number of patients die on the wards after discharge from intensive care units. Mortality after discharge from intensive care ranges from $9 \%$ to $27 \% .^{18}$ Our discharge triage model used objective data (age, end stage disease, physiology, length of stay, and cardiac surgery) in a logistic regression equation to identify patients at risk from inappropriate early discharge. We were able to do this because the database of the Riyadh ICU program captures daily data throughout a patient's stay in intensive care.

Among patients in the dataset we used to develop the model, those identified as at risk had a mortality of $14 \%$ compared with a mortality of only $1.5 \%$ among those not at risk. This is despite the low mortality after discharge from intensive care at Guy's of only 3.7\%. The model was applicable to the validation dataset despite a large difference in its mortality after discharge: $11.3 \%$ compared with $3.7 \%$ in the development dataset. The main difference between the two datasets was that the development dataset contained more patients who had undergone cardiac surgery. Eighteen out of the 19 other intensive care units did not treat patients who had undergone cardiac surgery. This disparity was accounted for by the cardiac surgery variable in the model.

By modelling a "what if" situation, whereby patients at risk and discharged on the same day were compared

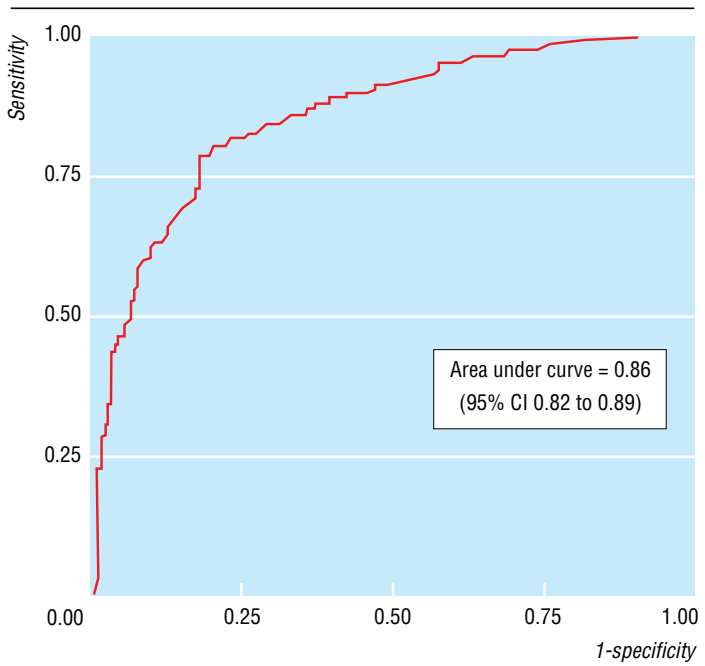

Fig 1 Receiver operator characteristic curve of discharge triage model

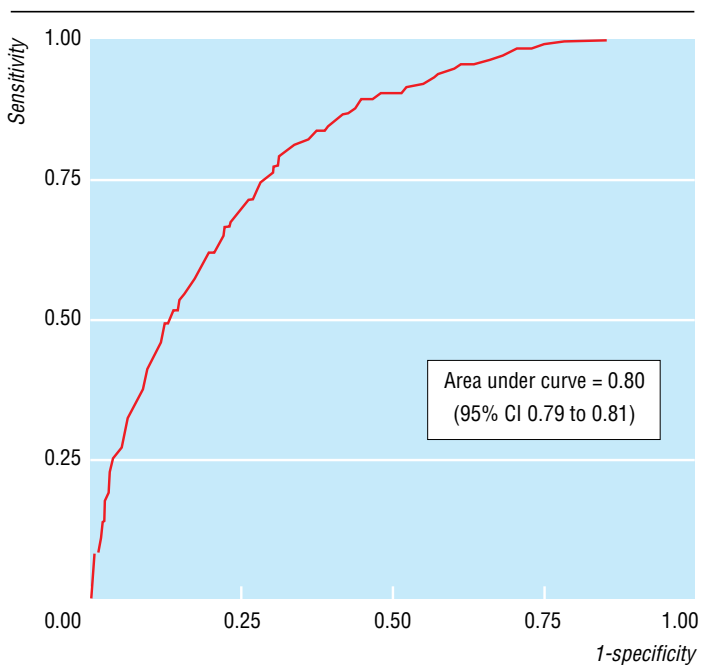

Fig 2 Receiver operator characteristic curve for validation dataset 


\section{What is already known on this topic}

In the United Kingdom, the mortality of patients who die on the ward after discharge from intensive care is unacceptably high (9\% to $27 \%)$

Indirect evidence has shown that this is due to too early and inappropriate discharge from intensive care that has increased over the past 10 years

\section{What this study adds}

A triage model identifies patients at risk from inappropriate discharge from intensive care

Mortality after discharge from intensive care may be reduced by $39 \%$ if these patients were to stay in intensive care for another 48 hours

An estimated $16 \%$ more beds are required if mortality after discharge from intensive care is to be reduced

with patients who stayed for another 24 to 48 hours, we showed a reduction in relative risk from 6.76 to 3.46 . Acute physiological points is the only variable in the model for which a reduction will lead to fall in the probability of dying on the ward. This variable is an aggregate of the weights of 12 physiological variables; normalisation of physiology will lead to a reduction in the variable and therefore a reduction in the probability of dying after discharge from intensive care.

\section{UK resources for intensive care}

The United Kingdom has limited resources allocated for the provision of intensive care facilities compared with many of its European counterparts, ${ }^{20}$ and regional differences in the number of available intensive care beds have been shown. ${ }^{21}$ Although the overall number of intensive care and high dependency beds has increased over the past 10 years, there has been a concurrent rise in hospital activity. ${ }^{22}$ Our modelling exercise suggests that up to $34 \%$ of patients are at risk and an increase of $16 \%$ in the number of intensive care beds is required to avoid deaths from inappropriate early discharges. Although this finding needs confirmation by a prospective study, it is consistent with the finding in the report by the Audit Commission in 1999 that up to $25 \%$ (with a median value of $5 \%$ ) of patients were still being discharged prematurely to allow more seriously ill patients to be admitted. ${ }^{24}$ Neither our discharge triage model nor discharge guidelines published by the Department of Health, ${ }^{25}$ which deal with the process of care, will have much impact until and unless the shortfall in provision of intensive care beds is corrected.

In summary, our model can provide additional information to help the doctor with the difficult problem of who to discharge from an intensive care unit to make room for a patient who needs urgent admission. The modelling exercise supports the findings of others that the provision of intensive care resources in the United Kingdom needs to be increased.

We thank members of the Riyadh ICU program users group for access to their database.

Contributors: KD (as part of her $\mathrm{PhD}$ ) and RWSC (PhD supervisor) collected data and were responsible for data pre-processing, development of the triage model, data analysis, and literature search. KD, RWSC, and RB wrote the paper jointly. RC will act as guarantor.

Funding: The Special Trustees of St Thomas's Hospital contributed to the funding of this project.

Competing interests: R W S Chang designed and developed the Riyadh ICU program and is a director of Medical Associated Software House, which markets the software.

1 Rowan KM, Kerr JH, Major E, McPherson K, Short A, Vessey MP. Intensive care society's APACHE II study in Britain and Ireland. I. Variations in case mix of adult admissions to general intensive care units and impact on outcome. BMJ 1993;307:972-6.

2 Rowan KM, Kerr JH, Major E, McPherson K, Short A, Vessey MP. Intensive care society's APACHE II study in Britain and Ireland. II. Outcome comparisons of intensive care units after adjustment for case mix by the American APACHE II method. BMJ 1993;307:977-81.

3 Bion J. Rationing intensive care. BMJ 1995;310:682-3.

4 Ryan DW. Providing intensive care. BMJ 1996;312:654.

5 Wallis CB, Davies HTO, Shearer AJ. Why do patients die on general ward after discharge from intensive care units? Anaesthesia 1997;52:9-14.

6 Dragstead L, Qvist J. Outcome from intensive care. A five-year study of 1308 patients: underlying causes of death. Eur J Anaesthesiol 1990;7:15968.

7 Ridley S, Purdie J. Cause of death after critical illness. Anaesthesi 1992;47:116-9.

8 Goldfrad C, Rowan K. Consequences of discharges from intensive care at night. Lancet 2000;355:1138-42.

9 Smith L, Orts CM, O’Neil I, Batchelor AM, Gascoigne AD, Baudouin SV. TISS and mortality after discharge from intensive care. Intensive Care Med 1999;25:1061-5.

10 Daly K, Bihari D. Multiple organ failure in the critically ill. Outcomes and costs - a six month follow up study. Intensive Care Med 1995;21(suppl 1):81

11 Knaus WA, Draper EA, Wagner WP, Zimmerman JE. APACHE II: a sever ity of disease classification system. Crit Care Med 1985;13:818-29.

12 Chang RWS, Jacobs S, Lee B. Predicting outcome of intensive care unit patients using computerised trend analysis of daily APACHE II scores corrected for organ system failure. Intensive Care Med 1988;14:558-66.

13 Cullen DJ, Keene R, Waternaux C, Kunsman JM, Caldera DL, Peterson H. Results, charges and benefits of intensive care for critically ill patients: update. Crit Care Med 1984;12:102-6.

14 SPSS. Neural connection 2.0 user's guide. Chicago: SPSS, 1997.

15 Lemeshow S, Hosmer DW. A review of goodness of fit statistics for use in the development of logistic regression models. Am J Epidemiol 1982;115:92-106.

16 Hanley JA, McNeil BJ. The meaning and use of the area under a receiver operator characteristic (ROC) curve. Radiology 1982;143:29-36.

17 Wasson JH, Sox HC, Neff RK, Goldman L. Clinical prediction rules. Applications and methodological standards. $N$ Engl J Med 1985;313:793-9.

18 Munn J, Willatts SM, Tooley MA. Health and activity after intensive care Anaesthesia 1995;50:1017-21.

19 Goldhill DR, Sumner A. Outcome of intensive care patients in a group of British intensive care units. Crit Care Med 1998:26:1337-45.

20 Vincent JL, Suter P, Bihari D, Bruining H. Organisation of intensive care units in Europe: lessons from the EPIC study. Intensive Care Med 1997;23:1181-4

21 Metcalf MA, McPherson K. Study of intensive care in England. London: Department of Heath, 1995.

22 Ridley SA, Burchett K, Burns A, Gunning K. A comparison of hospital and critical-care activity. Anaesthesia 1999;54:521-8.

23 Hensher M, Edwards N, Stokes R. International trends in the provision and utilisation of hospital care. BMJ 1999;319:845-8.

24 Audit Commission. Critical to success: the place of efficient and effective critical care services within the acute hospital. London: Audit Commission, 1999.

25 Working Group. Guidelines on admission to and discharge from intensive care and high dependency care units. London: Department of Health, 1996.

(Accepted 28 February 2001) 